\title{
Electron collisions with nitrous oxide
}

\author{
Carl Winstead and Vincent McKoy \\ A. A. Noyes Laboratory of Chemical Physics, California Institute of Technology, Pasadena, California 91125
}

(Received 4 December 1997)

\begin{abstract}
We have carried out theoretical studies of low-energy elastic electron collisions with nitrous oxide $\left(\mathrm{N}_{2} \mathrm{O}\right)$, obtaining differential, integral, and momentum-transfer cross sections. Polarization effects are incorporated in the electron-molecule scattering dynamics. A simple, objective, and physically motivated criterion is introduced for constructing a compact set of configurations that accurately accounts for polarization in resonant symmetries while avoiding overcorrelation. Our cross sections are in generally good agreement with experiment and with earlier high-level calculations, and most differences are readily understood. However, certain discrepancies between the calculated and measured differential cross sections remain puzzling. [S1050-2947(98)05905-8]
\end{abstract}

PACS number(s): $34.50 . \mathrm{Gb}$

\section{INTRODUCTION}

Low-energy elastic collisions between electrons and nitrous oxide, $\mathrm{N}_{2} \mathrm{O}$, have been the subject of several recent theoretical investigations [1-3]. Although the results of these investigations are generally in mutual qualitative agreement, there remain significant quantitative discrepancies both among the calculations and between the calculations and the measured total [4-9] and differential elastic [10-12] cross sections. Some of these discrepancies have well-understood origins. For example, both the position and the width of narrow shape resonances such as that found near $2.3 \mathrm{eV}$ in $\mathrm{N}_{2} \mathrm{O}$ are sensitive to aspects of the physics that have proven difficult to treat systematically in computational studies, and the resonance width is further affected by the neglect of vibrational motion in the calculations. However, other areas of disagreement-notably between the calculated [3] and measured $[10,11]$ differential elastic cross sections in the 5$10-\mathrm{eV}$ range - are less easily explained. There also remains some controversy regarding the existence of low-energy shape resonances of $\Sigma$ symmetry. Early analyses $[13,14]$ attributed the prominent $2.3-\mathrm{eV}$ resonance to a temporary anion of $\Sigma$ symmetry, and this assignment was accepted for some time [15,16]. A later study [17] modified this assignment, concluding that partially overlapping $\Sigma$ and $\Pi$ resonances are present. However, neither of the recent calculations that covered this energy range $[1,3]$ found evidence of a $\Sigma$ resonance there. Meanwhile, some experimental work has indicated that a broad $\Sigma$ resonance exists near $8 \mathrm{eV}$ $[17,18]$. However, only one of the two recent high-level calculations [1] finds such a feature near $8 \mathrm{eV}$; the other [3] places a $\Sigma$ resonance near $11 \mathrm{eV}$, while the more approximate calculation of Michelin et al. [2] yields a $\Sigma$ resonance at $13 \mathrm{eV}$ whose relation, if any, to the experimental feature at $8 \mathrm{eV}$ is unclear. In their recent measurements of the elastic differential cross section, Johnstone and Newell [11] found no evidence of such a resonance but did not rule out the presence of a weak, broad feature.

In light of the open issues identified above, further study of the elastic electron cross sections of $\mathrm{N}_{2} \mathrm{O}$ is warranted. The present paper describes calculations that we have carried out on elastic electron scattering by $\mathrm{N}_{2} \mathrm{O}$ using the Schwinger multichannel (SMC) method $[19,20]$. Like the
$R$-matrix studies of Sarpal et al. [1] and of Morgan et al. [3], the present SMC study incorporates polarization effects, which are vital to obtaining the correct behavior of the cross section at energies below $10 \mathrm{eV}$. We aim to improve on the previous studies, however, through a more systematic, physically motivated treatment of polarization that results in a better description both of resonant and of nonresonant or weakly resonant channels. As will be seen below, the SMC calculation reproduces very well the experimental location of the $\Pi$ resonance without any ad hoc "tuning" of the resonance position, and it clearly indicates the presence of a $\Sigma$ resonance near $8 \mathrm{eV}$. On the other hand, the differential cross sections in the $5-10-\mathrm{eV}$ range, though similar to previous $R$-matrix results [3], continue to differ substantially in form from the experimental cross sections [11,12], suggesting that some aspects of the collision physics will require a still more careful treatment. We discuss possible areas for future study below.

In the following section, we describe how the present calculations were carried out. Results are presented in Sec. III and discussed in Sec. IV. Our conclusions are summarized in Sec. V.

\section{THEORETICAL}

The SMC method has been described in detail elsewhere $[19,20,21]$. Thus we confine ourselves here to the particulars of the current calculations, especially our treatment of polarization and how it differs from earlier work.

The simplest widely useful treatment of low-energy electron-molecule scattering is the static-exchange approximation, i.e., the approximation in which the electron is scattered by Coulomb and (nonlocal) exchange potentials determined from the electron density of the isolated molecule. Because the static-exchange approximation omits the (net attractive) effect of the response of the molecular charge density to the presence of the projectile, it often performs poorly at the lowest energies and where resonances are present, in general placing shape resonances $2-4 \mathrm{eV}$ above their experimental locations and broadening them correspondingly. To obtain improved results, some accounting for polarization is necessary. In the SMC method, as in other $a b$ initio approaches [22,23], polarization is accounted for by 
including virtual excitations of the target (closed channels) among the $N$-electron molecular electronic wave functions from which the trial space of $(N+1)$-electron configurations is constructed. In general, the resulting $(N+1)$-electron configuration space will contain a partial representation both of polarization and of other forms of correlation, including correlation within the description of the target molecule. The resulting wave function is thus susceptible to "overcorrelation," particularly if, as is often the case, a simple HartreeFock wave function is used to represent the $N$-electron ground state of the molecule. The consequent overcorrection of the static-exchange resonance energies will place resonances below their experimental positions. A rigorous means of avoiding this problem is to use a high, or at least balanced, level of correlation for both target and $(N+1)$-electron spaces. Sarpal et al. [1] tested the use of a correlated target in their study of $\mathrm{N}_{2} \mathrm{O}$ and found an improved, but still too low, energy for the ${ }^{2} \Pi$ resonance, and much less satisfactory results for ${ }^{2} \Sigma$ symmetry. Their results may be attributable to the difficulty of obtaining a balanced description of $N$ - and $(N+1)$-electron correlation without the use of explicit criteria.

A far more efficient approach than introducing target correlation is to restrict the closed-channel configurations in the $(N+1)$-electron space to those that account most directly for polarization. As Schneider and Collins [24] noted in their seminal study of the polarization effects in scattering by $\mathrm{N}_{2}$, the target response in the resonant symmetry is mostly accounted for by "radial correlation," i.e., symmetrypreserving (and total-spin-preserving) excitations (in $\mathrm{N}_{2} \mathrm{O}$, ${ }^{1} \Sigma^{+}$states formed by $\sigma \rightarrow \sigma$ and $\pi \rightarrow \pi$ excitations). This observation is understood upon recognizing that the main polarization effect in the presence of a long-lived resonance is relaxation of the target orbitals toward anion orbitals during the lifetime of the quasibound state.

Morgan et al. [3] included only radial correlation in their study of $\mathrm{N}_{2} \mathrm{O}$ and noted that they obtained a better (higher) resonance position than did Sarpal et al. [1] in their uncorrelated-target study, which included both radial and angular (symmetry-changing) excitations. However, the resonance position obtained by Morgan et al. was still too low compared to experiment. It is apparent that employing all configurations of the form $(p \gamma \rightarrow q \gamma) n \pi$, where $p \gamma$ is an occupied orbital, $q \gamma$ a virtual orbital of the same symmetry, and $n \pi$ a $\pi$ virtual orbital, will include not only relaxation in the presence of the resonant $\pi$ orbital but also further correlation associated with nonresonant linear combinations of the $\pi$ virtuals. To ensure that the target and temporary-anion wave functions are of comparable quality, what we really desire is an accurate single-configuration representation of the resonant state, and nothing more. A procedure that we have found effective is to construct a valencelike virtual orbital $\tilde{\pi}$ that approximately represents the local portion of the resonance orbital and then to include only configurations of the form $(p \gamma \rightarrow q \gamma) \tilde{\pi}$ in our representation of polarization. This approach is quite analogous to the use of singleexcitation configuration interaction (SECI) to obtain excitedstate wave functions and energies that are of essentially single-configuration quality, since it likewise produces an essentially single-configuration description of the temporary anion using the ground-state target orbitals. Because both the
$N$-electron target wave function and the $(N+1)$-electron temporary anion wave function are of comparable quality, we have a foundation for expecting the energy difference (i.e., the resonance position) to be fairly accurate.

One simple way to construct $\widetilde{\pi}$ is to obtain the virtual orbitals as the eigenfunctions of a cation Fock operator subject to orthogonality constraints with respect to the occupied orbitals of the neutral target; here we are borrowing a technique commonly used to construct valencelike orbitals to initiate configuration interaction (CI) or multiconfiguration Hartree-Fock calculations [25]. In the present case, we employed a +2 cation Fock operator and obtained approximations to the resonance orbitals as the lowest-energy $\pi_{x}$ and $\pi_{y}$ eigenfunctions of that operator that could be constructed within the space of virtual orbitals obtained from the Hartree-Fock calculation on the $\mathrm{N}_{2} \mathrm{O}$ neutral. We emphasize that, although we found this particular procedure for constructing $\widetilde{\pi}$ simple and convenient, we fully expect that comparable results would be obtained with any of a number of alternative methods for constructing a valencelike approximate "resonance orbital."

In nonresonant channels, the polarization physics is completely different, as Schneider and Collins [24] observed, and a completely different approach to constructing virtual excitations is required. The most significant nonresonant polarization effect is the long-range interaction of the electron with the target, which, as we would expect on physical grounds, is most important at the lowest collision energies, and therefore primarily affects $s$-wave scattering in the totally symmetric representation $\left({ }^{2} \Sigma^{+}\right.$for $\left.\mathrm{N}_{2} \mathrm{O}\right)$. This longrange interaction is essentially that between a test charge (the projectile electron) and an induced dipole in the target molecule whose magnitude is determined by the polarizability of the target, and it thus is more deserving of the name "polarization" than the relaxation in the presence of a resonance that was discussed above. In contrast to the symmetrypreserving or radial virtual excitations needed for resonance relaxation, all excitations allowed by electric-dipole selection rules, including symmetry-breaking excitations, are needed to represent this polarization of the molecular charge density. Thus, for the ${ }^{2} \Sigma$ symmetry of $\mathrm{N}_{2} \mathrm{O}+e$, we must include closed-channel configurations of the form $(p \sigma$ $\rightarrow q \sigma) n \sigma, \quad\left(p \sigma \rightarrow q \pi_{x, y}\right) n \pi_{x, y}, \quad\left(p \pi_{x, y} \rightarrow q \sigma\right) n \pi_{x, y}$, $\left(p \pi_{x, y} \rightarrow q \pi_{x, y}\right) n \sigma, \quad\left(p \pi_{x, y} \rightarrow q \pi_{x, y}\right) n \delta_{x^{2}-y^{2}}, \quad$ and $\left(p \pi_{x, y}\right.$ $\left.\rightarrow q \delta_{x y}\right) n \pi_{y, x}$.

Because of the different physics in the nonresonant case-there is no quasibound state whose energy must be accurately determined relative to the neutral molecule'sovercorrelation of the $(N+1)$-electron space is not the major concern. Rather, because of the large number of configurations potentially required to represent the polarized-targetelectron system, it is undercorrelation that we must worry about: If we proceed naively to generate all closed-channel configurations of the dipole-allowed form just listed, we find that many thousands of configurations result for even modest-sized basis sets. In order to obtain a more manageable configuration space, we follow Rescigno and coworkers [22] by performing a linear transformation within the virtual-orbital space to construct a small set of polarized—or, more accurately, polarizing—orbitals defined so that polarization is accounted for by excitations into those 
orbitals alone. For a component $x_{\mu}$ of the dipole operator and occupied orbital $\sigma_{i}$, the polarizing orbital is defined as

$$
\phi_{i, \mu}=\sum_{j} \phi_{j} \frac{\left\langle\phi_{j}\left|x_{\mu}\right| \phi_{i}\right\rangle}{E_{j}-E_{i}},
$$

where the sum runs over virtual orbitals and $E_{j}$ and $E_{i}$ are orbital energies. Rescigno et al. obtain each polarizing orbital as the single non-null eigenvector of a rank-one operator; we prefer to construct the orbitals directly from the Hartree-Fock energies and the basis-set representation of the dipole transition operator. Also, Rescigno et al. obtain the virtual orbitals $\phi_{j}$ and corresponding energies $E_{j}$ from an $N$-particle excited-state Fock operator [26], while we employ the Hartree-Fock virtual orbitals and energies as in standard orbital perturbation theory [27]. In order to maintain an orthogonal set of virtual orbitals, we Schmidt-orthogonalize the polarizing orbitals constructed for different target orbitals among themselves, then Schmidt-orthogonalize the remaining virtual orbitals to the polarizing orbitals.

The basis set used throughout the calculation was the $6-311+\mathrm{G}(2 d)$ set internal to the electronic structure program GAMESS [28]. This basis set differs from the usual definition of $6-311+\mathrm{G}(2 d)[29,30]$ by including all six Cartesian components of the $d$ orbitals. With this basis, we obtain a Hartree-Fock energy of -183.736716 a.u. and a dipole moment of $0.631810 \mathrm{D}$ at the experimental equilibrium geometry, $r_{\mathrm{N}-\mathrm{N}}=1.128 \AA$ and $r_{\mathrm{N}-\mathrm{O}}=1.184 \AA$ [31]. These values compare quite well with previous results $(-183.73825$ a.u. and 0.6586 D) obtained at the HartreeFock level using a large basis set and the Hartree-Fock, rather than experimental, geometry [32]. However, it should be noted that the experimental value of the dipole moment, 0.1609 D [33], is much smaller than the Hartree-Fock value. Ordinarily, when the SMC and similar methods are applied to polar molecules, a correction for long-range scattering by the dipole potential must be added in order to obtain accurate cross sections, because neither the square-integrable representation of the wave function that the SMC method employs nor the small number of partial waves that other methods employ accounts adequately for such long-range scattering. In the present case, we omit this correction on the grounds that the true dipole moment is known to be so small. The effect on the calculated cross sections should be minor except at the very lowest energies and for near-forward scattering at higher energies. (Although, as has been pointed out from time to time $[34,35]$, fixed-nuclei elastic cross sections for polar molecules-or, more precisely, fixed-nuclei elastic scattering amplitudes in the partial-wave representationformally diverge at all angles when all partial waves are included, this divergence is unphysical, in that the adiabatic approximation [36] does not apply to very high partial waves. The contribution of these partial waves converges in a nonadiabatic treatment [37] because rotation of the molecule during the collision weakens the effective field [38]. The physically significant intermediate partial waves that are missing in our calculation do not contribute strongly to the cross section at intermediate angles. Thus our differential cross sections, and the integral and momentum-transfer cross sections derived from them, are meaningful. Similar remarks apply to the $R$-matrix results of Sarpal et al. and Morgan et al. [1,3], which employed partial-wave expansions truncated at $l=3$.)

For scattering in overall ${ }^{2} \Pi$ symmetry, a set of virtual excitations describing relaxation of the core within the temporary anion was constructed as described above, employing singlet excitations from each of the eight noncore occupied orbitals into all virtual orbitals having the same symmetry. After discarding a small number of partially redundant configurations in order to maintain the orthogonality of the $(N$ +1 )-electron configuration space, 416 doublet configuration state functions remained, divided equally between ${ }^{2} \Pi_{x}$ and ${ }^{2} \Pi_{y} ; 32$ of these were configurations of the form [core] $n \pi$, which would occur in a static-exchange calculation, and the remainder described polarization. Strictly, only one $\Pi$ component must be calculated, so that equivalent results could have been obtained using only 208 configuration state functions; however, since 416 configurations is still a quite small problem, we chose to treat ${ }^{2} \Pi_{x}$ and ${ }^{2} \Pi_{y}$ together for later convenience in calculating differential cross sections.

Scattering in ${ }^{2} \Sigma$ symmetry was treated with inclusion of singlet excitations into polarizing orbitals constructed for each of the eight noncore occupied orbitals. The construction procedure gives polarizing orbitals of mixed $\sigma$ and $\delta$ symmetry for certain excitations out of $1 \pi$ and $2 \pi$. Rather than decomposing these into good symmetry orbitals, we chose to treat ${ }^{2} \Delta$ scattering together with ${ }^{2} \Sigma$, though not to incorporate polarization for ${ }^{2} \Delta$, where it is unlikely to be important; we therefore included terms of the form [core] $n \delta$ as well as terms of the form [core] $n \sigma$ in the "static-exchange" portion of the configuration space. (The ${ }^{2} \Sigma$ and ${ }^{2} \Delta$ contributions can still be separated for interpretive purposes by partialwave decomposition of the scattering amplitude in the postprocessing phase.) Taking account of the facts that the Schmidt procedure does not mix higher-numbered orbitals into lower-numbered orbitals, that $\sigma \rightarrow \delta$ excitations do not contribute, and that the $\delta_{x y}$ polarizing orbitals generated from $p \pi_{x}$ and $p \pi_{y}$ are identical, 86 virtual excitations are required to obtain a complete description. The $(N+1)$-electron doublet configurations generated from these excitations, together with the static-exchange-type configurations, compose a space of 1813 configuration state functions. The scattering calculations were carried out on a CRAY T3D and on a HP Exemplar X-Class using our parallelized implementation of the SMC method [21].

\section{RESULTS}

The SMC integral elastic cross section, together with its decomposition into ${ }^{2} \Sigma,{ }^{2} \Pi$, and ${ }^{2} \Delta$ components, is presented in Fig. 1. In Fig. 2, the SMC integral cross section is compared to previous calculated results $[1-3]$ and to experimental total [7] and integral elastic $[10,11]$ cross sections. Differential cross sections are shown at selected energies in Figs. 3 and 4, along with measured values [10,11] where available. The calculated momentum-transfer cross section is compared in Fig. 5 to results derived from differential crosssection measurements $[10,11]$. Numerical values for the integral and momentum-transfer cross sections are given in Table I, and values of the differential cross section at selected energies are given in Table II. 


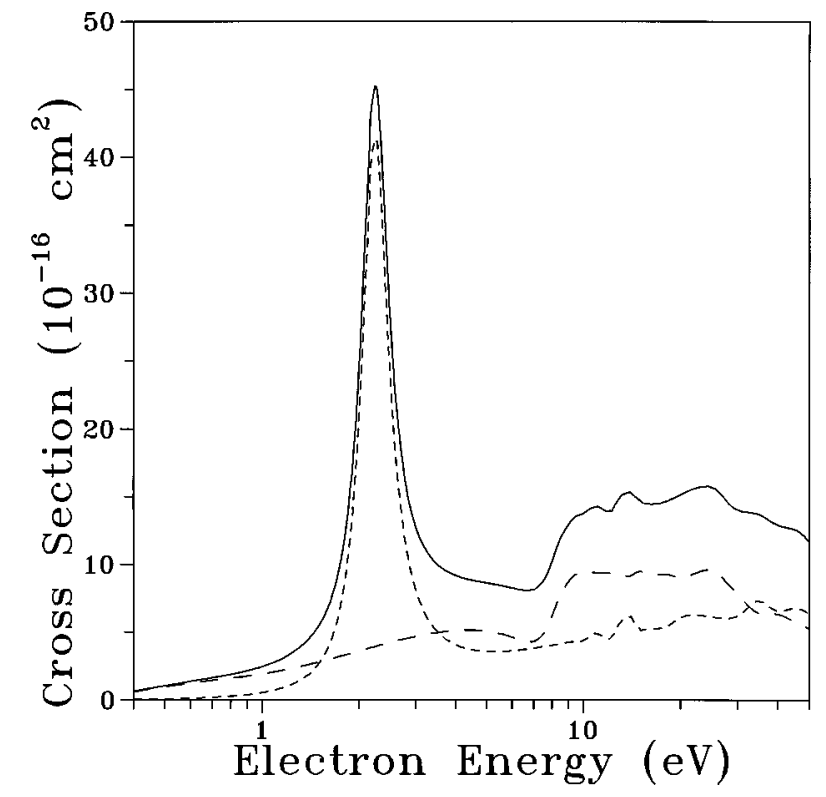

FIG. 1. Calculated integral elastic cross section for electron collisions with $\mathrm{N}_{2} \mathrm{O}$, together with its symmetry components. -----, ${ }^{2} \Pi$ component; - - - ${ }^{2} \Sigma+{ }^{2} \Delta$ component; - - summed integral cross section.

\section{DISCUSSION}

As Fig. 2 shows, the present SMC calculation is very successful at reproducing the experimental position of the low-energy ${ }^{2} \Pi$ resonance. As we have discussed in Sec. II, this success is not attributable to the SMC method per se, but rather to the balanced way in which we have treated polarization. In contrast, earlier calculations that employed a simi-

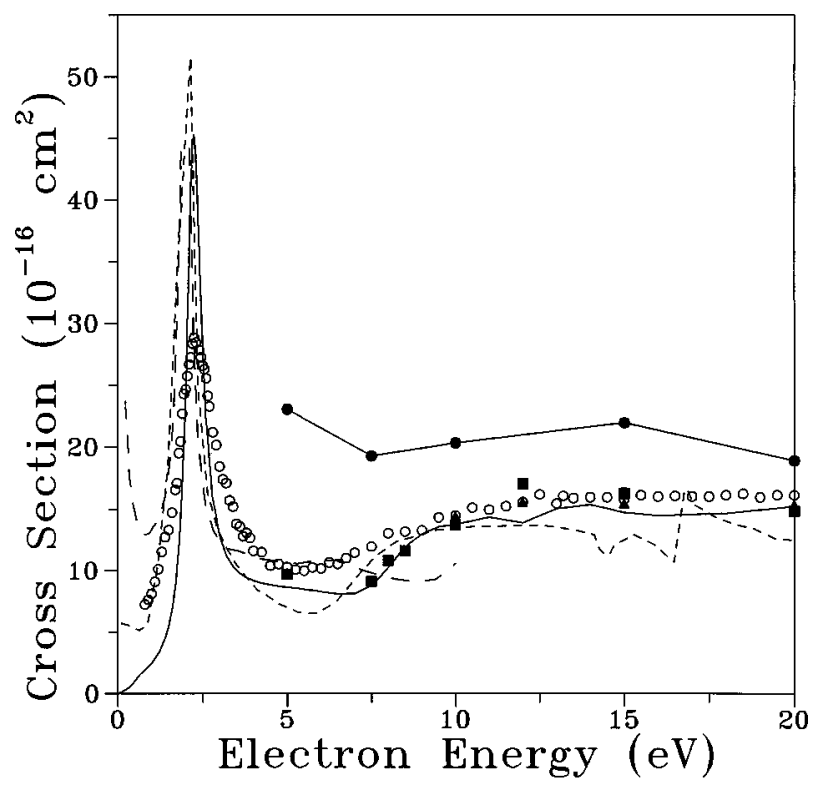

FIG. 2. Integral elastic and total electron cross sections for $\mathrm{N}_{2} \mathrm{O}$. Calculated elastic cross sections are -----, Sarpal et al., Ref. [1]; --- , Morgan et al., Ref. [3]; -•-•-, Michelin et al., Ref. [2]; present work. Measured elastic cross sections are from Johnstone and Newell, Ref. [11] (squares) and Marinković et al., Ref. [10] (triangles). The open circles are the total cross section measured by Szmytkowski et al., Ref. [7]. lar closed-channel description of polarization [1,3], lacking clear physical criteria for constructing the closed-channel space, tended to overcorrelate the resonant symmetry and thus to place the resonance too low in energy. The most successful of these previous calculations for the resonant symmetry was, as we might expect, that of Sarpal et al. [1] employing CI descriptions of both the target and the ( $N$ $+1)$-electron spaces; however, even in that case, the resonance position is somewhat too low, and much less satisfactory results were obtained in ${ }^{2} \Sigma$ symmetry.

We find that the ${ }^{2} \Pi$ eigenphase sum can be fitted very well to the Breit-Wigner form, $\tan ^{-1}\left[\Gamma / 2\left(E_{r}-E\right)\right]$, plus a low-order polynomial representing the background, if we employ $2.19 \mathrm{eV}$ for the resonance position $E_{r}$ and $0.52 \mathrm{eV}$ for the width $\Gamma$ (Fig. 6). However, the cross-section maximum occurs at about $2.25 \mathrm{eV}$, in excellent agreement with its experimental location, rather than at $2.19 \mathrm{eV}$. This shift arises through interference between resonant and background scattering; indeed, as seen in Fig. 7 , the ${ }^{2} \Pi$ contribution to the cross section does not have a pure Breit-Wigner form, but does fit quite well to a Fano [39] profile,

$$
\sigma(E)=\sigma_{0}(E) \frac{\left[q+\left(E-E_{r}\right) / \frac{1}{2} \Gamma\right]^{2}}{1+\left[\left(E-E_{r}\right) / \frac{1}{2} \Gamma\right]^{2}},
$$

using the above values for $E_{r}$ and $\Gamma$ together with $q=10.5$ and the simple choice $\sigma_{0}(E)=0.17 E \AA^{2}(E$ in $\mathrm{eV})$.

Because our calculation, like the earlier calculations [13], was carried out in a fixed-nuclei approximation at the equilibrium nuclear geometry, the resonance width that we obtain is too small, and the maximum value of the cross section, about $45 \AA^{2}$, is too large compared with experiment. Dubé and Herzenberg [16] extracted a resonance width of $0.7 \mathrm{eV}$ from their analysis of the vibrational excitation cross sections [15], while the maximum value of the total cross scattering cross section is about $29 \AA^{2}$ [7]. Previous calculations $[1,3]$ using a comparable level of approximation gave maximum values even larger than ours due to the expected narrowing of the resonance when it is shifted to lower energy. When configuration interaction was included in the description of the target, Sarpal et al. [1] obtained a value of $0.65 \pm 0.1 \mathrm{eV}$ for the width. However, the maximum value of the cross section remained too large $\left(51.6 \AA^{2}\right)$. Since, moreover, we expect further broadening to result from a consideration of vibrational motion, the apparent agreement between the CI and experimental widths may be fortuitous.

Below the resonance energy, the cross section at first decreases rapidly. However, as the electron energy becomes small, one eventually expects the cross section to increase, due to efficient scattering of very slow electrons by the dipole potential. Thus there should be a minimum in the cross section somewhere between 0 and $2.3 \mathrm{eV}$, and in fact such a minimum is observed in the total cross section at about 0.4 $\mathrm{eV}[5,6,9]$. Both of the earlier calculations that extended to this range exhibit a minimum followed by an upturn in this cross section as the energy goes to zero, though at rather different energies, in one case lower [1] and in the other case higher [3] than experiment. Our own calculation, which omits the long-range dipole interaction, does not reproduce these features. 

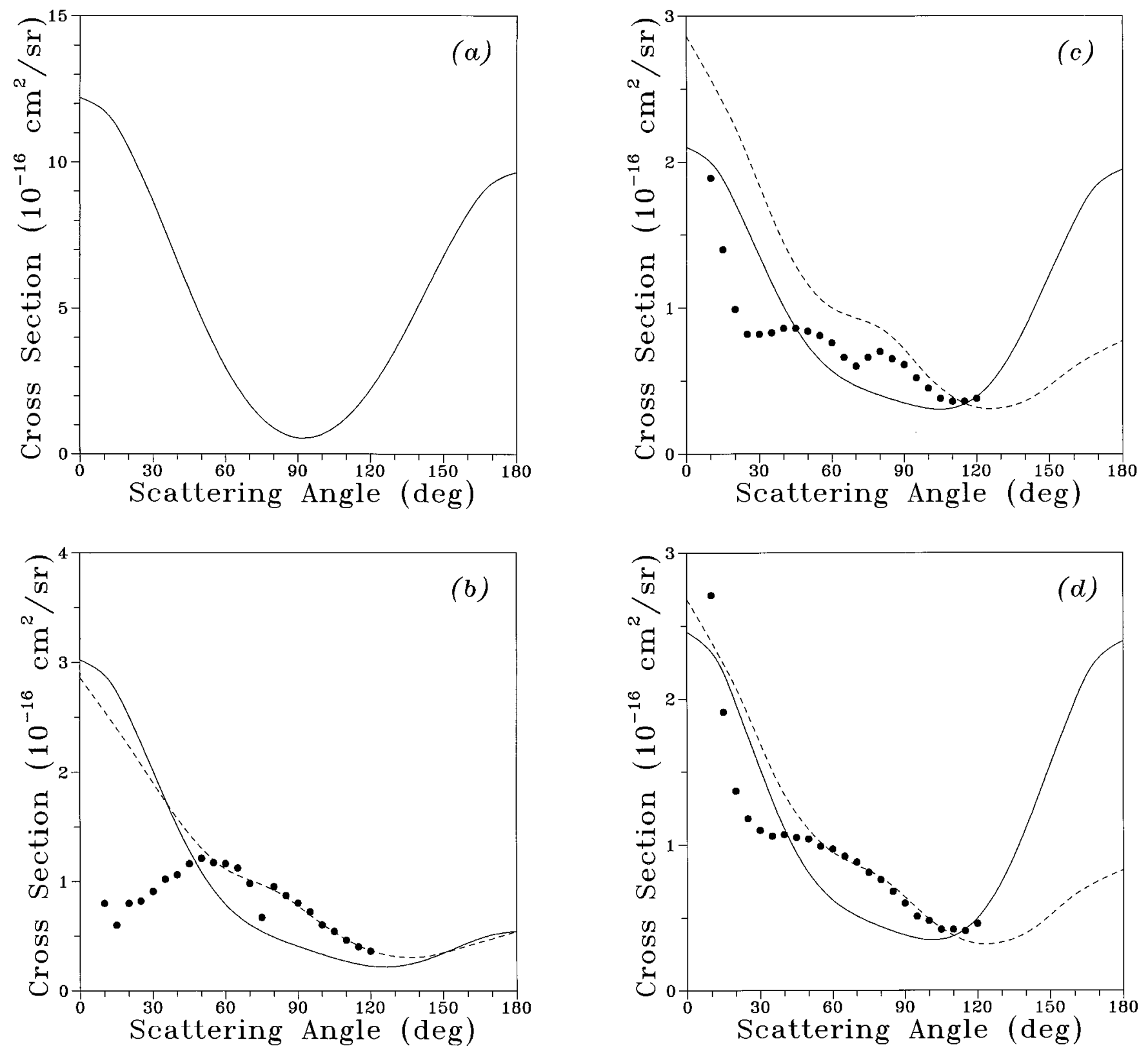

FIG. 3. Differential elastic cross sections for electron scattering by $\mathrm{N}_{2} \mathrm{O}$ at (a) $2.25 \mathrm{eV}$, (b) $5 \mathrm{eV}$, (c) $7.5 \mathrm{eV}$, and (d) $8 \mathrm{eV}$. -, Measurements of Johnstone and Newell, Ref. [11]; -----, calculation of Morgan et al., Ref. [3]; - - , present calculation.

Turning to the $8-\mathrm{eV}$ region, we find, in agreement with Sarpal et al. [1] and with experiment [17,18], that there is a rather broad and weak ${ }^{2} \Sigma$ resonance present. Sarpal et al. obtained values of approximately $8 \mathrm{eV}$ and approximately 2 $\mathrm{eV}$ for the position and width, respectively. From a fit to the ${ }^{2} \Sigma+{ }^{2} \Delta$ eigenphase sum, shown in Fig. 8, we extract a resonance position $E_{r}=8.0 \mathrm{eV}$ and width $\Gamma=2.8 \mathrm{eV}$. For comparison, the vibrational-excitation measurement of Andric and Hall [17] showed a peak at $8.3 \mathrm{eV}$ whose width, as estimated from their Fig. 9, is slightly greater than $3 \mathrm{eV}$.

Although the calculation of Morgan et al. [3] did not produce a resonance in the vicinity of $8 \mathrm{eV}$, it did place a ${ }^{2} \Sigma$ resonance near $11 \mathrm{eV}$, intermediate between $8 \mathrm{eV}$ and the energy, $13 \mathrm{eV}$, of a ${ }^{2} \Sigma$ resonance found in the staticexchange approximation $[1,2]$. It thus appears reasonable to conclude that, on the one hand, the calculation of Morgan et al. includes a somewhat incomplete representation of polarization effects in ${ }^{2} \Sigma$ symmetry, and, on the other hand, that the feature at $13 \mathrm{eV}$ in the static-exchange results does indeed correlate with the $8-\mathrm{eV}$ resonance. The $5-\mathrm{eV}$ shift in the position of this resonance due to polarization is, in our experience, unusually large.

Johnstone and Newell [11] searched for the 8-eV $\Sigma$ resonance in the differential elastic cross section, taking measurements at closely spaced energies $(7.5,8.0$, and $8.5 \mathrm{eV}$ ) within the appropriate range, but found no clear evidence of it. They concluded that the resonance must be too broad and weak to stand out against the nonresonant background. Our results (Figs. 3 and 4) indicate that the form of the cross section is in fact little affected by the resonance over the range of angles that was accessible in the experiment, but that the backscattering cross section is strongly affected; the resonance therefore stands out more clearly in the calculated momentum-transfer cross section (Fig. 5) than in the integral elastic cross section (Fig. 1).

Above $10 \mathrm{eV}$, the integral elastic cross section contains 

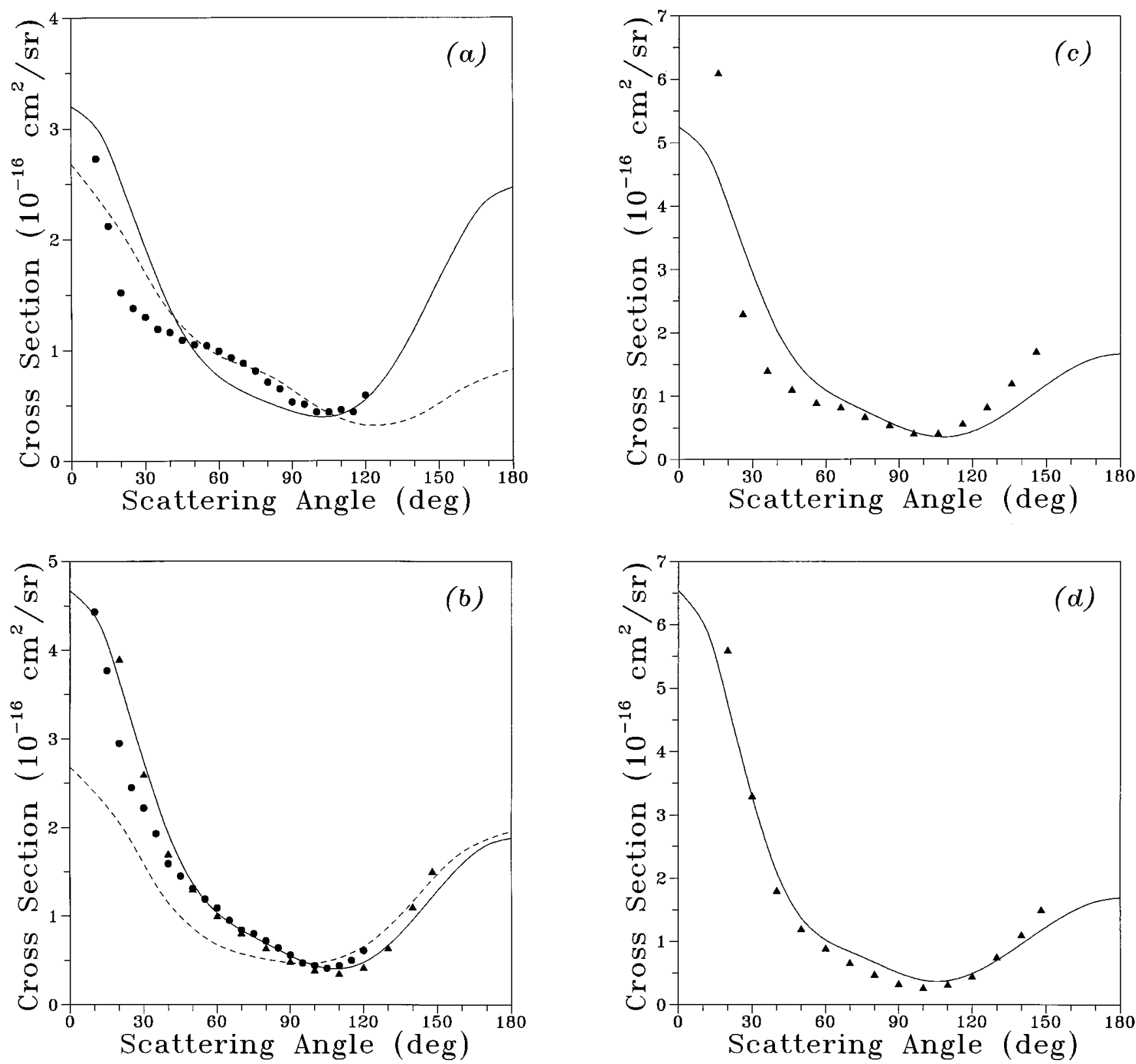

FIG. 4. As in Fig. 3, at (a) $8.5 \mathrm{eV}$, (b) $10 \mathrm{eV}$, (c) $12 \mathrm{eV}$, and (d) $15 \mathrm{eV}$, with the addition of data from Marinković et al., Ref. [10] (triangles).

structure that is not fully resolved on our energy mesh, particularly in ${ }^{2} \Pi$ symmetry from 10 to $20 \mathrm{eV}$ (Figs. 1 and 2). This structure is attributable to spurious resonances that arise because some of the "closed channel" terms included in the description of polarization are in fact open channels at these impact energies. Such spurious structure is expected above the first electronic excitation threshold in calculations of this kind. Similar behavior is seen in the calculation of Sarpal et al. [1] at these energies.

Overall the magnitude of our integral elastic cross section is in quite good agreement with the experimental elastic cross section $[10,11]$ and with the $R$-matrix calculations [1,3]. The results of Michelin et al. [2] are consistently larger. The discrepancy is not attributable to their use of the static-exchange approximation, which should work quite well at higher energies; we found the static-exchange cross section to have virtually the same magnitude as the staticexchange-plus-polarization cross section at $10 \mathrm{eV}$ and above, as did Sarpal et al. [1]. It may instead arise from the treatment of scattering by the long-range dipole potential. Michelin et al. employ a closure procedure to include the contribution of high partial waves to the scattering amplitude within the dipole-Born approximation. However, as discussed in Sec. II, including all partial wave contributions in fixed-nuclei elastic cross section calculations for polar molecules leads to divergent cross sections. Absent a careful (nonadiabatic) treatment, a calculation truncated at lower partial waves may give more reliable results for weakly polar molecules like $\mathrm{N}_{2} \mathrm{O}$.

Although the agreement between our integral elastic cross section and the experimental integral cross section is generally quite good (Fig. 2), there are marked discrepancies between our differential cross sections and the measured values. We expect the calculation to underestimate forward scattering since, as noted in Sec. II, it includes no long-range dipole component. The absence of this component, together 


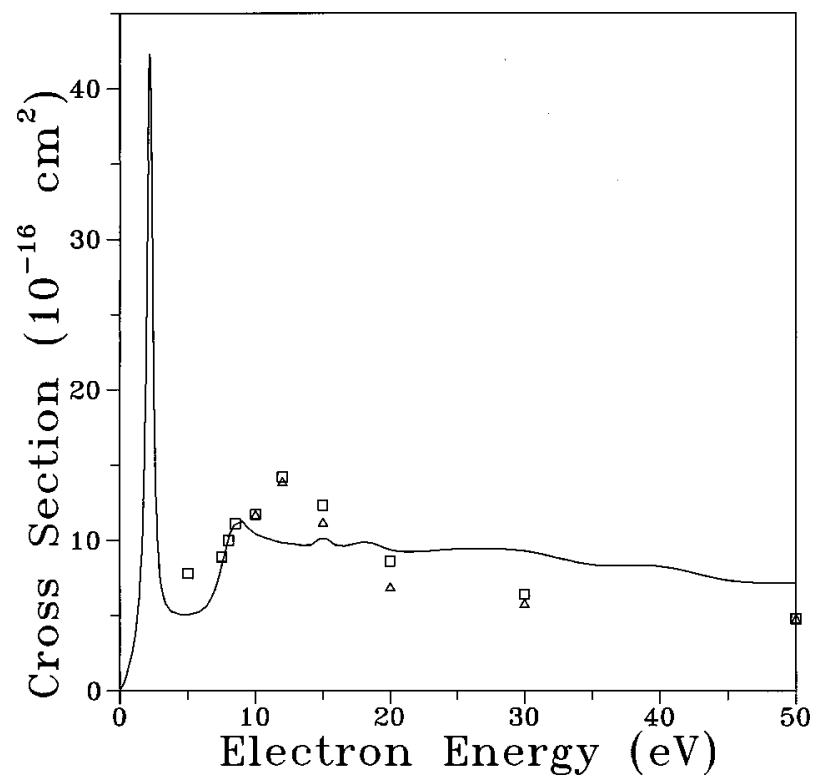

FIG. 5. Momentum-transfer cross section. Experimentally derived values are from Refs. [10] (triangles) and [11] (squares); the line is the present calculation.

with basis-set limitations, may explain the growing disagreement at small angles between our calculated cross section and experiment as the energy increases above $10 \mathrm{eV}$ (Fig. 4). In the 5-10-eV range, however, there is a serious qualitative disagreement between the calculated and experimental differential cross sections. The cross sections of Morgan et al. [3], which also differ qualitatively from experiment in this energy range, on the whole resemble our results, though differing in detail, as would be expected due to the shift in location of the ${ }^{2} \Sigma$ resonance. As Morgan et al. noted, the disagreement between theory and experiment at these energies is not easily explained. Because two independent experiments $[11,12]$ gave substantially similar results, we must tentatively conclude that one or more of the approximations common to the calculations is unexpectedly poor at these energies. The most likely candidates are the singleconfiguration Hartree-Fock approximation for the target wave function and the fixed-nuclei approximation. The latter is particularly suspect because this energy range lies within the tail of the $2.3-\mathrm{eV}$ resonance, whose effects on the cross section may be particularly sensitive to nuclear motion; moreover, the experimental cross sections show rapid variations with angle suggestive of interference phenomena not properly represented in the calculations. At present we cannot test the quality of the Hartree-Fock approximation because our SMC code does not yet accommodate a CI representation of the target. Sarpal et al. [1], who did employ a CI target wave function, unfortunately did not report differential cross sections. We can test the quality of the fixed-nuclei approximation by incorporating nuclear motion within the adiabatic approximation [36], performing multiple fixednuclei calculations that sample the geometries accessible via the zero-point motion of the nuclei and averaging the resulting cross sections together with appropriate weights. We plan to explore the effects of such vibrational averaging in future work.

The agreement between the experimental momentum transfer cross sections and our calculation, Fig. 5, though
TABLE I. Integral and momentum-transfer cross sections.

\begin{tabular}{|c|c|c|c|c|c|}
\hline \multirow[b]{2}{*}{$\begin{array}{l}\text { Energy } \\
(\mathrm{eV})\end{array}$} & \multicolumn{2}{|c|}{$\begin{array}{l}\text { Cross section } \\
\left(10^{-16} \mathrm{~cm}^{2}\right)\end{array}$} & \multirow[b]{2}{*}{$\begin{array}{c}\text { Energy } \\
(\mathrm{eV})\end{array}$} & \multicolumn{2}{|c|}{$\begin{array}{l}\text { Cross section } \\
\left(10^{-16} \mathrm{~cm}^{2}\right)\end{array}$} \\
\hline & Integral & $\begin{array}{c}\text { Momentum } \\
\text { Transfer }\end{array}$ & & Integral & $\begin{array}{l}\text { Momentum } \\
\text { Transfer }\end{array}$ \\
\hline 0.01 & 0.02 & 0.02 & 4.0 & 9.17 & 5.16 \\
\hline 0.1 & 0.13 & 0.17 & 4.2 & 9.00 & 5.10 \\
\hline 0.2 & 0.27 & 0.34 & 4.4 & 8.88 & 5.07 \\
\hline 0.4 & 0.65 & 0.76 & 4.6 & 8.78 & 5.06 \\
\hline 0.6 & 1.37 & 1.46 & 4.8 & 8.70 & 5.05 \\
\hline 0.8 & 1.89 & 2.17 & 5.0 & 8.63 & 5.06 \\
\hline 1.0 & 2.45 & 3.02 & 5.2 & 8.56 & 5.08 \\
\hline 1.2 & 3.26 & 4.24 & 5.4 & 8.49 & 5.12 \\
\hline 1.4 & 4.52 & 6.06 & 5.6 & 8.42 & 5.17 \\
\hline 1.5 & 5.46 & 7.34 & 5.8 & 8.34 & 5.24 \\
\hline 1.6 & 6.79 & 9.10 & 6.0 & 8.26 & 5.34 \\
\hline 1.7 & 8.74 & 11.53 & 6.5 & 8.09 & 5.78 \\
\hline 1.8 & 11.80 & 15.07 & 7.0 & 8.11 & 6.71 \\
\hline 1.85 & 13.97 & 17.46 & 7.5 & 8.75 & 8.28 \\
\hline 1.9 & 16.74 & 20.38 & 8.0 & 10.23 & 10.07 \\
\hline 1.95 & 20.25 & 23.91 & 8.5 & 11.92 & 11.08 \\
\hline 2.0 & 24.63 & 28.07 & 9.0 & 12.95 & 11.27 \\
\hline 2.05 & 29.83 & 32.68 & 9.5 & 13.56 & 10.80 \\
\hline 2.1 & 35.49 & 37.23 & 10.0 & 13.74 & 10.42 \\
\hline 2.15 & 40.74 & 40.80 & 11.0 & 14.32 & 10.09 \\
\hline 2.2 & 44.34 & 42.29 & 12.0 & 13.84 & 9.81 \\
\hline 2.25 & 45.30 & 41.10 & 13.0 & 14.98 & 9.75 \\
\hline 2.3 & 43.57 & 37.60 & 14.0 & 15.33 & 9.64 \\
\hline 2.35 & 40.04 & 32.90 & 15.0 & 14.67 & 10.18 \\
\hline 2.4 & 35.81 & 28.06 & 16.0 & 14.45 & 9.65 \\
\hline 2.5 & 27.98 & 20.09 & 18.0 & 14.62 & 9.90 \\
\hline 2.6 & 22.30 & 14.86 & 20.0 & 15.15 & 9.37 \\
\hline 2.8 & 15.88 & 9.54 & 25.0 & 15.75 & 9.41 \\
\hline 3.0 & 12.84 & 7.30 & 30.0 & 14.01 & 9.29 \\
\hline 3.2 & 11.24 & 6.25 & 35.0 & 13.72 & 8.36 \\
\hline 3.4 & 10.33 & 5.71 & 40.0 & 12.89 & 8.27 \\
\hline 3.6 & 9.77 & 5.42 & 45.0 & 12.60 & 7.34 \\
\hline 3.8 & 9.41 & 5.25 & 50.0 & 11.69 & 7.18 \\
\hline
\end{tabular}

reasonably good, is somewhat less satisfactory than the agreement for the integral cross sections extracted from the same measured and calculated differential cross section data. Unlike the integral cross section, the momentum transfer cross section depends strongly on large-angle scattering that in fact has not been measured, but rather extrapolated from measurements at smaller angles. The small magnitude and slow variation of the differential cross section at these intermediate angles make an accurate extrapolation to high angles difficult. We believe that the disagreements below $20 \mathrm{eV}$ in Fig. 5 arise primarily from uncertainties in extrapolation of the measurements. At higher energies the same extrapolation uncertainties enter, but an incomplete representation of high partial waves in the calculation may also be a factor. As just discussed, an indication that high partial-wave contributions may be underrepresented at higher energies is found at nearforward angles, where the computed differential cross sec- 
TABLE II. Selected differential elastic cross sections.

\begin{tabular}{rrrrrr}
\hline \hline & \multicolumn{5}{c}{$\begin{array}{c}\text { Differential cross section } \\
\left(10^{-16} \mathrm{~cm}^{2} / \mathrm{sr}\right)\end{array}$} \\
$\begin{array}{rrrrrr}\text { Angle } \\
\text { (deg) }\end{array}$ & $2.2 \mathrm{eV}$ & $5 \mathrm{eV}$ & $8 \mathrm{eV}$ & $10 \mathrm{eV}$ & $15 \mathrm{eV}$ \\
\hline 0 & 11.08 & 3.02 & 2.46 & 4.67 & 6.54 \\
10 & 10.68 & 2.88 & 2.32 & 4.39 & 6.04 \\
20 & 9.56 & 2.51 & 1.97 & 3.65 & 4.77 \\
30 & 7.94 & 2.01 & 1.52 & 2.75 & 3.29 \\
40 & 6.11 & 1.51 & 1.11 & 1.94 & 2.09 \\
50 & 4.32 & 1.08 & 0.81 & 1.37 & 1.37 \\
60 & 2.78 & 0.79 & 0.62 & 1.03 & 1.02 \\
70 & 1.60 & 0.60 & 0.51 & 0.84 & 0.83 \\
80 & 0.86 & 0.49 & 0.44 & 0.69 & 0.67 \\
90 & 0.57 & 0.41 & 0.38 & 0.55 & 0.50 \\
100 & 0.74 & 0.33 & 0.35 & 0.44 & 0.39 \\
110 & 1.36 & 0.27 & 0.37 & 0.40 & 0.38 \\
120 & 2.39 & 0.22 & 0.50 & 0.48 & 0.49 \\
130 & 3.76 & 0.22 & 0.74 & 0.67 & 0.70 \\
140 & 5.37 & 0.26 & 1.11 & 0.95 & 0.96 \\
150 & 7.02 & 0.34 & 1.55 & 1.28 & 1.23 \\
160 & 8.49 & 0.44 & 1.97 & 1.58 & 1.46 \\
170 & 9.51 & 0.51 & 2.28 & 1.80 & 1.63 \\
180 & 9.88 & 0.54 & 2.39 & 1.88 & 1.69 \\
\hline \hline
\end{tabular}

tion is smaller than the measurements. It is conceivable that the same contributions could decrease the computed differential cross section at large angles through destructive interference and thereby decrease the computed momentum transfer cross section.

\section{CONCLUSION}

We have studied low-energy elastic electron scattering by $\mathrm{N}_{2} \mathrm{O}$ in the fixed-nuclei, static-exchange plus polarization ap-

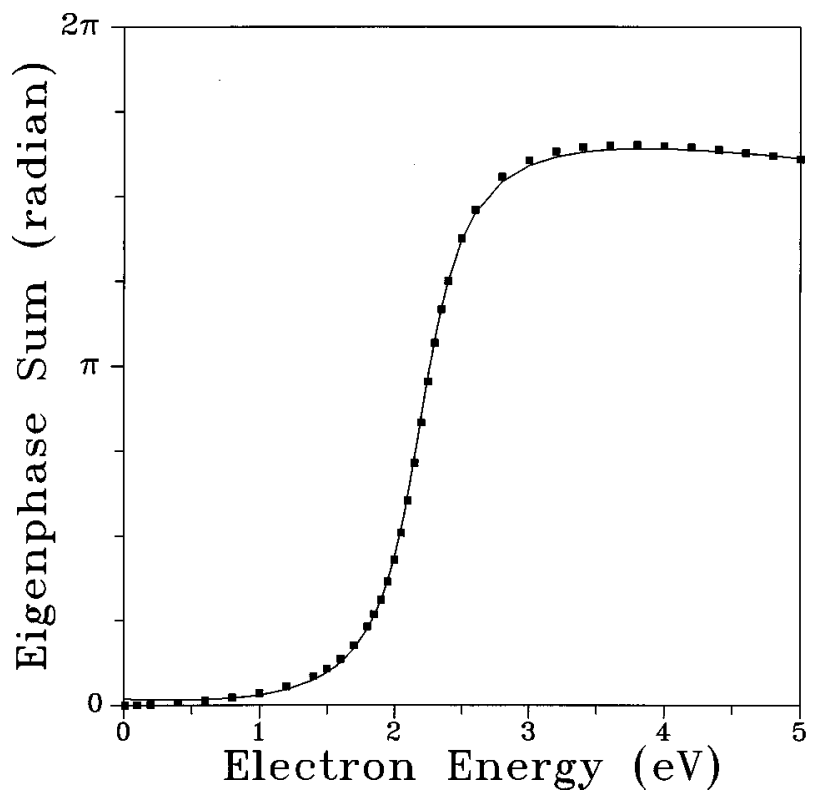

FIG. 6. Eigenphase sum in ${ }^{2} \Pi$ symmetry near the resonance. The squares are the calculated points; the line is a fit to $\Delta(E)=$ $-0.18-0.15 E-0.004 E^{2}+2 \tan ^{-1}[0.26 /(2.19-E)]$. (The factor of 2 in the arctangent term arises from the twofold degeneracy of the resonance.)

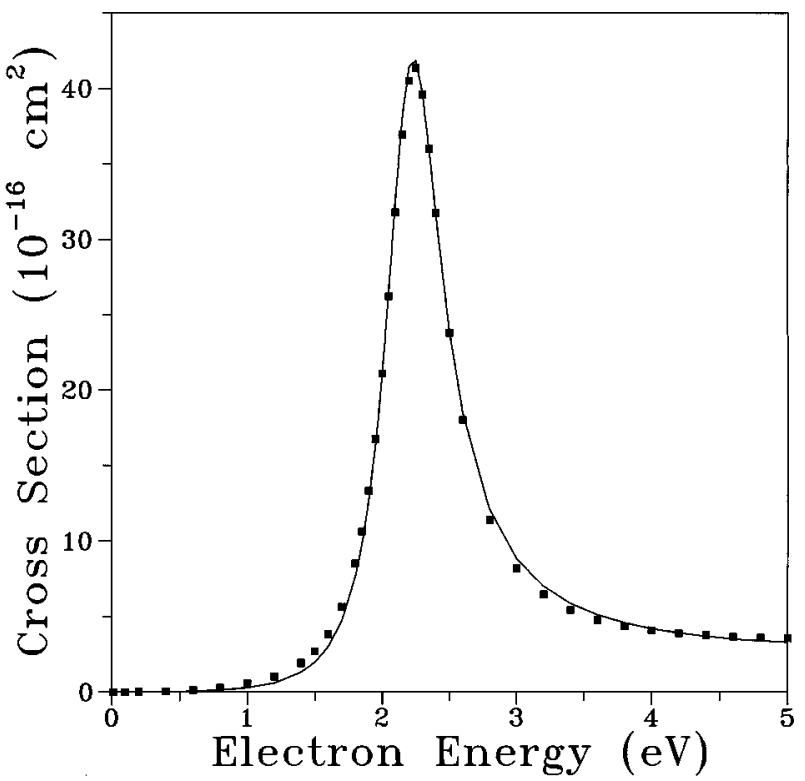

FIG. 7. Fano profile fit for the ${ }^{2} \Pi$ component of the cross section. The squares are the calculated points; the line is the fit (see text for discussion).

proximation. By using objective, physically motivated criteria for choosing the appropriate representation of polarization effects, we obtain improved results in comparison to earlier, otherwise similar calculations $[1,3]$. In particular, we find that incorporating only those virtual excitations necessary to produce an accurate single-configuration description of the temporary anion yields a position for the ${ }^{2} \Pi$ resonance in excellent agreement with experiment. In ${ }^{2} \Sigma$ symmetry, we concur with experiment $[17,18]$ and with one earlier calculation [1] in placing a broad resonance near $8 \mathrm{eV}$. Because this resonance is broad and rather weak, and because it influences the cross section most strongly in the

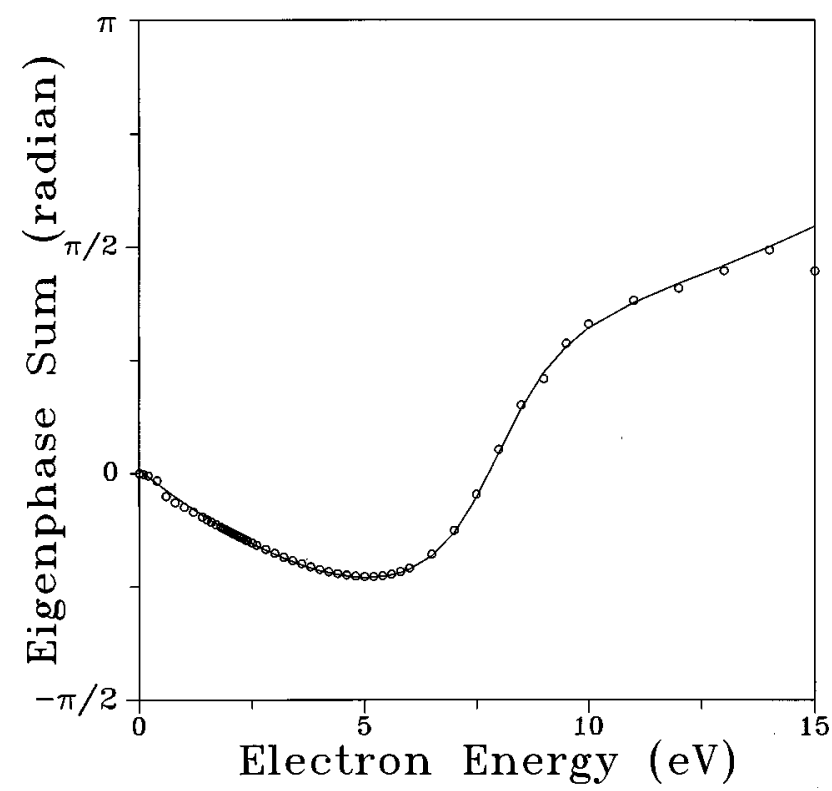

FIG. 8. Eigenphase sum in ${ }^{2} \Sigma+{ }^{2} \Delta$ symmetry. The circles are the calculated points; the line is a fit to $\Delta(E)=-0.15-0.27 E$ $+0.132 E^{2}+\tan ^{-1}[1.42 /(7.95-E)]$. 
near-forward and near-backward directions, it is not readily observed in the differential elastic cross section [11]. The location of the ${ }^{2} \Sigma$ resonance appears to be especially sensitive to polarization effects; it occurs at $11 \mathrm{eV}$ in the calculation of Morgan et al. [3] and at $13 \mathrm{eV}$ in the static-exchange approximation $[1,2]$. The shape of the differential elastic cross section between 5 and $10 \mathrm{eV}$ requires further investigation. Neither our calculation nor that of Morgan et al. produces results that resemble the observed $[11,12]$ cross section in this energy range. The influence of zero-point vibrational motion, or of target correlation, may be important in this case.

\section{ACKNOWLEDGMENTS}

These calculations made use of the CRAY T3D operated by the JPL-Caltech Supercomputing Project, which was supported in part by NASA's Mission to Planet Earth, and of the HP Exemplar X-Class operated by the Center for Advanced Computing Research, which was supported in part by the National Science Foundation. Financial support by the U.S. Department of Energy, Office of Basic Energy Sciences, under Grant No. DE-FG03-97ER14814, and by the National Science Foundation through the project "Parallel I/O Methodologies for I/O Intensive Grand Challenge Applications" is gratefully acknowledged.
[1] B. K. Sarpal, K. Pfingst, B. M. Nestmann, and S. D. Peyerimhoff, J. Phys. B 29, 857 (1996); 29, 1877(E) (1996).

[2] S. E. Michelin, T. Kroin, and M.-T. Lee, J. Phys. B 29, 2115 (1996); 29, 4319(E) (1996).

[3] L. A. Morgan, C. J. Gillan, J. Tennyson, and X. Chen, J. Phys. B 30, 4087 (1997).

[4] E. Brüche, Ann. Phys. (Leipzig) 83, 1065 (1927).

[5] C. Ramsauer and R. Kollath, Ann. Phys. (Leipzig) 7, 176 (1930).

[6] A. Zecca, I. Lazzizzera, M. Krauss, and C. E. Kuyatt, J. Chem. Phys. 61, 4560 (1974).

[7] C. Szmytkowski, G. Karwasz, and K. Maciąg, Chem. Phys. Lett. 107, 481 (1984).

[8] C. Szmytkowski, K. Maciąg, G. Karwasz, and D. Filipović, J. Phys. B 22, 525 (1989).

[9] C. K. Kwan, Y.-F. Hsieh, W. E. Kauppila, S. J. Smith, T. S. Stein, M. N. Uddin, and M. S. Dababneh, Phys. Rev. Lett. 52, 1417 (1984).

[10] B. Marinković, C. Szmytkowski, V. Pejčev, D. Filipović, and L. Vušković, J. Phys. B 19, 2365 (1986).

[11] W. M. Johnstone and W. R. Newell, J. Phys. B 26, 129 (1993).

[12] M. Kubo, D. Matsunaga, T. Suzuki, and H. Tanaka, in Proceedings of the 12th International Conference on the Physics of Electronic and Atomic Collisions, edited by S. Datz (NorthHolland, Amsterdam, 1981), p. 360; as quoted in Refs. [10, $11]$.

[13] P. J. Chantry, J. Chem. Phys. 51, 3369 (1969).

[14] J. N. Bardsley, J. Chem. Phys. 51, 3384 (1969).

[15] R. Azria, S. F. Wong, and G. J. Schulz, Phys. Rev. A 11, 1309 (1975)

[16] L. Dubé and A. Herzenberg, Phys. Rev. A 11, 1314 (1975).

[17] L. Andrić and R. I. Hall, J. Phys. B 17, 2713 (1984).

[18] M. Tronc, L. Malegat, R. Azria, and Y. Le Coat, in Proceedings of the 12th International Conference on the Physics of Electronic and Atomic Collisions (Ref. [12]), p. 372.
[19] K. Takatsuka and V. McKoy, Phys. Rev. A 24, 2473 (1981).

[20] K. Takatsuka and V. McKoy, Phys. Rev. A 30, 1734 (1984).

[21] C. Winstead and V. McKoy, Adv. At., Mol., Opt. Phys. 36, 183 (1996).

[22] T. N. Rescigno, B. H. Lengsfield, and C. W. McCurdy, in Modern Electronic Structure Theory, edited by D. F. Yarkony (World Scientific, Singapore, 1995), Pt. I, p. 501.

[23] C. J. Gillan, O. Nagy, P. G. Burke, L. A. Morgan, and C. J. Noble, J. Phys. B 20, 4585 (1987).

[24] B. I. Schneider and L. A. Collins, Phys. Rev. A 30, 95 (1984).

[25] C. W. Bauschlicher, J. Chem. Phys. 72, 880 (1980).

[26] W. J. Hunt and W. A. Goddard, Chem. Phys. Lett. 3, 414 (1969).

[27] For example, A. Szabo and N. S. Ostlund, Modern Quantum Chemistry (Macmillan, New York, 1982), p. 338ff.

[28] M. W. Schmidt, K. K. Baldridge, J. A. Boatz, S. T. Elbert, M. S. Gordon, J. H. Jensen, S. Koseki, N. Matsunaga, K. A. Nguyen, S. J. Su, T. L. Windus, M. Dupuis, and J. A. Montgomery, J. Comput. Chem. 14, 1347 (1993).

[29] R. Krishnan, J. S. Binkley, R. Seeger, and J. A. Pople, J. Chem. Phys. 72, 650 (1980).

[30] M. J. Frisch, J. A. Pople, and J. S. Binkley, J. Chem. Phys. 80, 3265 (1984).

[31] G. Herzberg, Molecular Spectra and Molecular Structure (Van Nostrand, New York, 1966), Vol. III, p. 596.

[32] W. D. Allen, Y. Yamaguchi, A. G. Csaszar, D. A. Clabo, R. B. Remington, and H. F. Schaefer, Chem. Phys. 145, 427 (1990).

[33] J. M. L. J. Reinartz, W. L. Meerts, and A. Dymanus, Chem. Phys. 31, 19 (1978).

[34] W. R. Garrett, Phys. Rev. A 4, 2229 (1971).

[35] T. N. Rescigno, B. H. Lengsfield, C. W. McCurdy, and S. D. Parker, Phys. Rev. A 45, 7800 (1992).

[36] D. M. Chase, Phys. Rev. 104, 838 (1956).

[37] A. M. Arthurs and A. Dalgarno, Proc. R. Soc. London, Ser. A 256, 540 (1960).

[38] W. R. Garrett, Mol. Phys. 24, 465 (1972).

[39] U. Fano, Phys. Rev. 124, 1866 (1961). 